\title{
IMPROVED COMPACT VISIBILITY REPRESENTATION OF PLANAR GRAPH VIA SCHNYDER'S REALIZER*
}

\author{
CHING-CHI LIN ${ }^{\dagger}$, HSUEH-I LU ${ }^{\dagger}$, AND I-FAN SUN ${ }^{\dagger}$
}

\begin{abstract}
Let $G$ be an $n$-node planar graph. In a visibility representation of $G$, each node of $G$ is represented by a horizontal line segment such that the line segments representing any two adjacent nodes of $G$ are vertically visible to each other. In the present paper we give the best known compact visibility representation of $G$. Given a canonical ordering of the triangulated $G$, our algorithm draws the graph incrementally in a greedy manner. We show that one of three canonical orderings obtained from Schnyder's realizer for the triangulated $G$ yields a visibility representation of $G$ no wider than $\left\lfloor\frac{22 n-40}{15}\right\rfloor$. Our easy-to-implement $O(n)$-time algorithm bypasses the complicated subroutines for four-connected components and four-block trees required by the best previously known algorithm of Kant. Our result provides a negative answer to Kant's open question about whether $\left\lfloor\frac{3 n-6}{2}\right\rfloor$ is a worst-case lower bound on the required width. Also, if $G$ has no degree-three (respectively, degreefive) internal node, then our visibility representation for $G$ is no wider than $\left\lfloor\frac{4 n-9}{3}\right\rfloor$ (respectively, $\left\lfloor\frac{4 n-7}{3}\right\rfloor$ ). Moreover, if $G$ is four-connected, then our visibility representation for $G$ is no wider than $n-1$, matching the best known result of Kant and He. As a by-product, we give a much simpler proof for a corollary of Wagner's theorem on realizers due to Bonichon, Le Saëc, and Mosbah.
\end{abstract}

Key words. visibility representation, planar graph algorithm, graph drawing, realizer, canonical ordering

AMS subject classifications. 05C62, 05C85, 68W35, 68U05, 68R10, 94C15

DOI. $10.1137 / \mathrm{S} 0895480103420744$

1. Introduction. In a visibility representation of a planar graph $G$, the nodes of $G$ are represented by nonoverlapping horizontal line segments, called node segments, such that the node segments representing any two adjacent nodes of $G$ are vertically visible to each other. (See Figure 1.1.) Computing compact visibility representations of planar graphs is not only fundamental in algorithmic graph theory [31, 9] but also practically important in VLSI layout design [27].

Without loss of generality the input $G$ can be assumed to be an $n$-node plane triangulation. Following the convention of placing the endpoints of node segments on the grid points, one can easily see that any visibility representation of $G$ can be made no higher than $n-1$. Otten and van Wijk [25] gave the first known algorithm for visibility representations of planar graphs, but no width bound was provided for the output. Rosenstiehl and Tarjan [26], Tamassia and Tollis [30], and Nummenmaa [24] independently proposed $O(n)$-time algorithms whose outputs are no wider than $2 n-5$. Kant $[15,17]$ improved the required width to at most $\left\lfloor\frac{3 n-6}{2}\right\rfloor$ by decomposing $G$ into its four-connected components and then combining the visibility representations of the four-connected components into a visibility representation of $G$. Kant left open the question of whether the upper bound $\left\lfloor\frac{3 n-6}{2}\right\rfloor$ on the width is also a worst-case lower bound. In the present paper we provide a negative answer to Kant's question by

* Received by the editors January 4, 2003; accepted for publication (in revised form) November 22, 2003; published electronically July 2, 2004. A preliminary version of this paper appeared in Proceedings of the 20th Annual Symposium on Theoretical Aspects of Computer Science, H. Alt and M. Habib, eds., Lecture Notes in Comput. Sci. 2607, Springer-Verlag, Berlin, 2003, pp. 14-25.

http://www.siam.org/journals/sidma/18-1/42074.html

${ }^{\dagger}$ Institute of Information Science, Academia Sinica, Taiwan, Republic of China (hil@iis.sinica. edu.tw, www.iis.sinica.edu.tw/ ${ }^{\mathrm{hil} /} /$ ). The research of the second author was supported in part by NSC grants NSC-91-2213-E-001-028 and NSC-92-2213-E-001-006. 


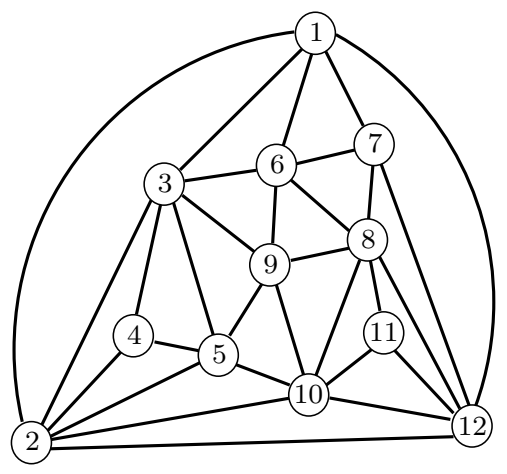

(a)

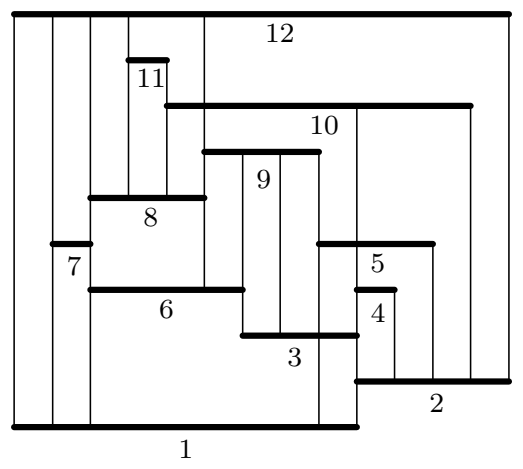

(b)

FIG. 1.1. A plane triangulation and one of its visibility representations.

presenting an algorithm that always produces a visibility representation for $G$ whose width is at most $\left\lfloor\frac{22 n-40}{15}\right\rfloor$.

Our algorithm, just like that of Nummenmaa [24], is based upon the concept of canonical ordering for plane triangulations. Specifically, our algorithm draws $G$ incrementally in a greedy manner according to any given canonical ordering of $G$. An arbitrary canonical ordering of $G$ may yield a visibility representation with width $2 n-$ $O(1)$. Rosenstiehl and Tarjan [26] even conjectured that selecting a node ordering to minimize the area of the corresponding visibility representation is NP-hard. We show that the required width can be bounded by $\left\lfloor\frac{22 n-40}{15}\right\rfloor$ using the best one out of the three canonical orderings obtained from Schnyder's realizer [29, 28] for $G$. Our algorithm can easily be implemented to run in $O(n)$ time, bypassing the complicated subroutines of finding four-connected components and four-block trees [14] required by the best previously known algorithm of Kant $[15,17]$. Also, for the case that $G$ has no degreethree (respectively, degree-five) internal node, the output visibility representation of our algorithm is no wider than $\left\lfloor\frac{4 n-9}{3}\right\rfloor$ (respectively, $\left\lfloor\frac{4 n-7}{3}\right\rfloor$ ). Moreover, for the case that $G$ is four-connected, the output visibility representation of our algorithm is no wider than $n-1$, matching the best known result due to Kant and He [18, 19].

Schnyder's realizer $[29,28]$ for plane triangulation was invented for obtaining compact straight-line drawing of plane graphs. Researchers $[5,7,8,11,12,13,16,19]$ also obtained similar and other graph-drawing results using the concept of canonical ordering for triconnected plane graphs. Nakano [23] attempted to explain the hidden relation between these two concepts. Recently, Chiang, Lin, and Lu [4] presented a new algorithmic tool, the orderly spanning tree, that extends the concept of st-ordering [10] (respectively, canonical ordering and realizer) for plane graphs not required to be biconnected (respectively, triconnected and triangulated). The orderly spanning tree has been successfully applied to obtain improved results in compact graph drawing $[4,20,3]$, succinct graph encoding with query support $[4,6]$, and design of compact routing tables [22]. Recently, Bonichon, Gavoille, and Hanusse [1] obtained the best known upper bounds on the numbers of distinct labeled and unlabeled planar graphs based on the well orderly spanning tree, a special case of the orderly spanning tree. As a matter of fact, we first successfully obtained the results of this paper using the orderly spanning tree and then found out that Schnyder's realizer suffices. 
Our analysis requires an equality (see Lemma 2.3) relating the number of internal nodes in the three trees of a realizer $R$ of $G$ and the number of faces of $G$ intersecting with all three trees of $R$. The equality was proved very recently by Bonichon, Le Saëc, and Mosbah [2] as a corollary of the so-called Wagner's theorem [32] on Schnyder's realizers. Their proof requires a careful case analysis for 32 different configurations. As a by-product, we give a much simpler proof for the equality without relying on Wagner's theorem on realizers.

The remainder of the paper is organized as follows. Section 2 gives the preliminaries. Section 3 describes and analyzes our algorithm. Section 4 discusses the tightness of our analysis. Section 5 concludes the paper with an open question.

2. Preliminaries. Let $G$ be the input $n$-node plane triangulation, a planar graph equipped with a fixed planar embedding such that the boundary of each face is a triangle. Clearly, $G$ has $2 n-5$ internal faces. Let $I$ consist of the internal nodes of $G$. Let $R=\left(T_{1}, T_{2}, T_{3}\right)$ be a realizer of $G$, which is obtainable in $O(n)$ time [28, 29]. That is, the following properties hold for $R$ :

- The internal edges of $G$ are partitioned into three edge-disjoint trees $T_{1}, T_{2}$, and $T_{3}$, each rooted at a distinct external node of $G$.

- The neighbors of each node $v$ in $I$ form six blocks $U_{1}, D_{3}, U_{2}, D_{1}, U_{3}$, and $D_{2}$ in counterclockwise order around $v$, where $U_{j}$ (respectively, $D_{j}$ ) consists of the parent (respectively, children) of $v$ in $T_{j}$ for each $j \in\{1,2,3\}$.

For each index $i \in\{1,2,3\}$, let $\ell_{i}$ be the node labeling of $G$ obtained from the counterclockwise preordering of the spanning tree $\bar{T}_{i}$ of $G$ consisting of $T_{i}$ plus the two external edges of $G$ that are incident to the root of $T_{i}$. (Each $\bar{T}_{i}$ is, as a matter of fact, an orderly spanning tree [4] of $G$.) Let $\ell_{i}(v)$ be the label of $v$ with respect to $\ell_{i}$. For example, Figure 2.1 shows a realizer of the plane triangulation shown in Figure 1.1(a) with labeling $\ell_{1}$. The counterclockwise preordering of $\bar{T}_{2}$ is $2,12,10,11,5,9,4,3,6,8,7,1$; and that of $\bar{T}_{3}$ is $12,1,7,8,11,10,9,6,3,5,4,2$.

Lemma 2.1 (see, e.g., $[4,24,6]$ ). The following properties hold for each index $i \in\{1,2,3\}$, where $u_{1}$ and $u_{2}$ are the nodes with $\ell_{i}\left(u_{1}\right)=1$ and $\ell_{i}\left(u_{2}\right)=2$.

1. The subgraph $G_{k}$ of $G$ induced by the nodes $v$ with $1 \leq \ell_{i}(v) \leq k$ is biconnected. The boundary of $G_{k}$ 's external face is a cycle $C_{k}$ containing $u_{1}$ and $u_{2}$.

2. If $v$ is the node with $\ell_{i}(v)=k$, then $v$ is on $C_{k}$, and the neighbors of $v$ in $G_{k-1}$ form an interval with at least two nodes on the path $C_{k-1}-\left\{\left(u_{1}, u_{2}\right)\right\}$.

3. The neighbors of $v$ in $G$ form the following four blocks in counterclockwise order around $v$ : (1) the parent of $v$ in $T_{i}$, (2) the node set consisting of the neighbors $u$ in $G-T_{i}$ with $\ell_{i}(u)<\ell_{i}(v)$, (3) the children of $v$ in $T_{i}$, and (4) the node set consisting of the neighbors $u$ in $G-T_{i}$ with $\ell_{i}(u)>\ell_{i}(v)$.

A labeling $\ell$ of $G$ that labels the external nodes by 1,2, and $n$ and satisfies Lemmas 2.1(1) and (2) is a canonical ordering of $G$ (e.g., see [24, 16, 8]). Therefore, $\ell_{1}, \ell_{2}$, and $\ell_{3}$ are all canonical orderings of $G$.

For each node $v$ of $G$, let $\operatorname{deg}(v)$ denote the degree of $v$, i.e., the number of neighbors of $v$ in $G$. For each index $i \in\{1,2,3\}$, let $\operatorname{deg}_{i}^{-}(v)$ (respectively, $\operatorname{deg}_{i}^{+}(v)$ ) be the number of neighbors $u$ of $v$ in $G$ with $\ell_{i}(u)<\ell_{i}(v)$ (respectively, $\ell_{i}(u)>\ell_{i}(v)$ ). Clearly, we have $\operatorname{deg}(v)=\operatorname{deg}_{i}^{-}(v)+\operatorname{deg}_{i}^{+}(v)$. For each node $v$ in $I$, let

$$
\begin{aligned}
\operatorname{score}_{i}(v) & =\min \left\{\operatorname{deg}_{i}^{+}(v), \operatorname{deg}_{i}^{-}(v)\right\} ; \\
\operatorname{score}(v) & =\operatorname{score}_{1}(v)+\operatorname{score}_{2}(v)+\operatorname{score}_{3}(v) .
\end{aligned}
$$




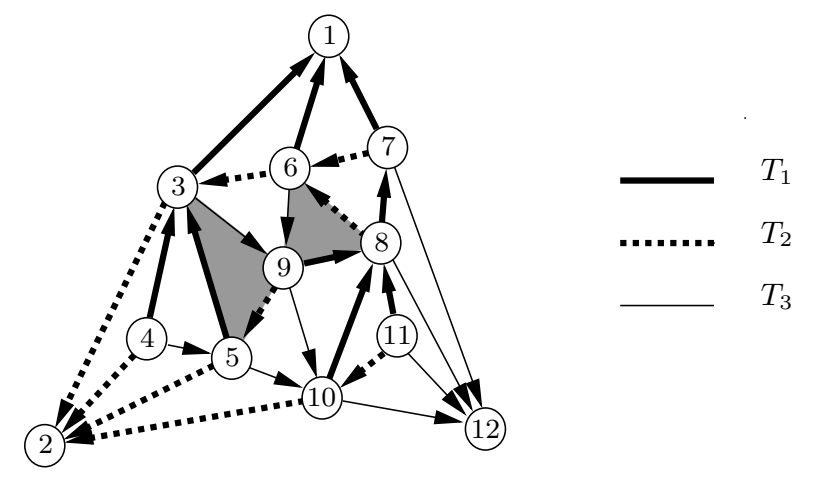

FIG. 2.1. A realizer for the plane triangulation shown in Figure $1.1(\mathrm{a})$, where $(3,5,9)$ and $(6,9,8)$ are the only two cyclic faces with respect to this realizer. The orientation of each edge is from a child to its parent in the corresponding tree.

For example, if $\ell_{1}$ is the labeling obtained from the tree $T_{1}$ consisting of the thick edges shown in Figure 2.1, then we have $\operatorname{score}_{1}\left(v_{8}\right)=2, \operatorname{score}_{1}\left(v_{9}\right)=1, \operatorname{score}_{1}\left(v_{10}\right)=2$, and $\operatorname{score}_{1}\left(v_{11}\right)=1$. Let

$$
\operatorname{score}_{i}=\sum_{v \in I} \operatorname{score}_{i}(v)
$$

Let $[\pi]$ be 1 (respectively, 0 ) if condition $\pi$ is true (respectively, false). Let $L_{i}$ consist of the leaves of $T_{i}$. For each node $v \in I$, let

$$
\operatorname{int}(v)=\sum_{i=1}^{3}\left[v \notin L_{i}\right] .
$$

Let $B$ consist of the internal nodes $v$ of $G$ with $\operatorname{int}(v)=2$ and $\operatorname{deg}(v)=5$. We have the following lemma.

Lemma 2.2. For each node $v$ in $I$, we have $\operatorname{score}(v) \geq 3+2 \cdot \operatorname{int}(v)-[v \in B]$.

Proof. By the definition of realizer and Lemma 2.1(3), it is clear that

$$
\begin{aligned}
& \operatorname{score}_{1}(v)=\min \left\{\left|D_{3}\right|+2,\left|D_{1}\right|+\left|D_{2}\right|+1\right\} ; \\
& \operatorname{score}_{2}(v)=\min \left\{\left|D_{1}\right|+2,\left|D_{2}\right|+\left|D_{3}\right|+1\right\} ; \\
& \operatorname{score}_{3}(v)=\min \left\{\left|D_{2}\right|+2,\left|D_{1}\right|+\left|D_{3}\right|+1\right\} .
\end{aligned}
$$

We may assume without loss of generality that $\left|D_{1}\right| \geq\left|D_{2}\right| \geq\left|D_{3}\right| \geq 0$. One can verify the lemma by examining the inequality for all possible values $\{0,1,2,3\}$ of $\operatorname{int}(v)$. For example, if $v \in B$, then we know score $(v)=2+2+2=6$ by $\left|D_{1}\right|=\left|D_{2}\right|=1$ and $\left|D_{3}\right|=0$. Also, if $\operatorname{int}(v)=2$ and $v \notin B$, then we have score $(v) \geq 2+2+3=7$ by observing $\left|D_{1}\right| \geq 2,\left|D_{2}\right| \geq 1$, and $\left|D_{3}\right|=0$. The other cases can be verified similarly.

An internal face of $G$ is cyclic if its boundary intersects with all three trees $T_{1}, T_{2}$, and $T_{3}$. An internal face of $G$ is acyclic if it is not cyclic. For example, in Figure 2.1, faces $(3,5,9)$ and $(6,9,8)$ are cyclic; all the other internal faces are acyclic. Let $c$ be the number of cyclic faces of $G$. The following lemma was recently proved by Bonichon, Le Saëc, and Mosbah [2] in an equivalent form. Our alternative proof is much simpler. 


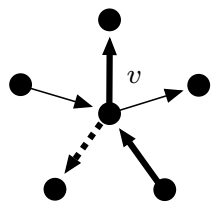

(a)

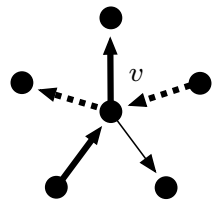

(b)

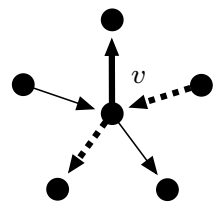

(c)

FIG. 2.2. Three different kinds of nodes $v$ in $B$.

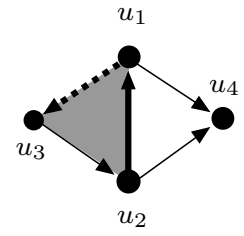

(a)

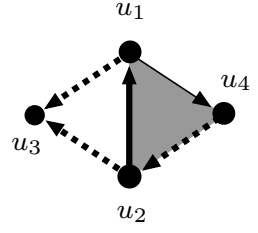

(b)

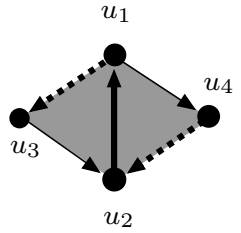

(c)

FIG. 2.3. If $u_{1}$ and $u_{2}$ are two nodes of $B$ that are adjacent in $G$, then at least one of faces $\left(u_{1}, u_{2}, u_{3}\right)$ and $\left(u_{1}, u_{2}, u_{4}\right)$ is cyclic.

Lemma 2.3 (see [2]). $\sum_{v \in I} \operatorname{int}(v)=n+c-4$.

Proof. For each index $i \in\{1,2,3\}$, let int ${ }_{i}$ be the number of internal nodes in $T_{i}$. Clearly, $\sum_{v \in I} \operatorname{int}(v)=\sum_{i=1}^{3} \operatorname{int}_{i}-3$. For each node $v \in I$, let $p_{i}(v)$ denote the parent of $v$ in $T_{i}$. For each $v \in L_{i}$, one can verify that $F_{i}(v)=\left(v, p_{j}(v), p_{k}(v)\right)$ is an acyclic face of $G$, where $\{i, j, k\}=\{1,2,3\}$. By the orientations of the three edges, one can see that for each acyclic face $F$, there is exactly one pair $(i, v)$ such that $v \in L_{i}$ and $F=F_{i}(v)$. Since $G$ has $2 n-5$ internal faces, the bijection between leaves and acyclic faces shows $\sum_{i=1}^{3}\left|L_{i}\right|=2 n-c-5$. Therefore, $\sum_{i=1}^{3} \operatorname{int}_{i}=3(n-2)-(2 n-c-5)=$ $n+c-1$.

LEMMA 2.4.

1. If $G$ has no degree-three internal nodes, then $\sum_{i=1}^{3} \operatorname{score}_{i} \geq 5 n-15$.

2. If $G$ has no degree-five internal nodes, then $\sum_{i=1}^{3}$ score $_{i} \geq 5 n-17$.

3. If $G$ is unrestricted, then $\sum_{i=1}^{3}$ score $_{i} \geq \frac{23 n}{5}-16$.

Proof. By Lemma 2.2 we know that if node $v$ in $I$ has degree more than 3 , then score $(v) \geq 5$. By $|I|=n-3$, statement 2.4 holds. It follows from Lemmas 2.2 and 2.3 that $\sum_{i=1}^{3} \operatorname{score}_{i}=\sum_{v \in I} \operatorname{score}(v) \geq \sum_{v \in I} 3+2 \cdot \operatorname{int}(v)-[v \in B]=3(n-3)+2(n+$ $c-4)-|B|$. Therefore, $\sum_{i=1}^{3}$ score $_{i} \geq 5 n+2 c-|B|-17$, which implies that (a) statement 2.4 holds (by observing that each node of $B$ has degree five in $G$ ), and (b) statement 2.4 can be proved by ensuring $|B|-2 c \leq \frac{2 n}{5}-1$ as follows.

Let $k$ be the number of connected components in the subgraph $G[B]$ of $G$ induced by $B$. Since each of those $2 n-5$ internal faces of $G$ is incident to at most one connected component $G[B]$, and each connected component in $G[B]$ is incident to at least five internal faces of $G$, we have $5 k \leq 2 n-5$.

Let $u_{1}$ and $u_{2}$ be two adjacent nodes of $B$ such that $\left(u_{1}, u_{2}\right)$ is an incoming edge of $u_{1}$. (That is, $u_{1}$ is the parent of $u_{2}$ in some tree $T_{i}$ of $R$.) Let $\left(u_{3}, u_{1}, u_{2}\right)$ and $\left(u_{4}, u_{1}, u_{2}\right)$ be the two faces of $G$ that contain edge $\left(u_{1}, u_{2}\right)$. One can see that at least one of faces $\left(u_{1}, u_{2}, u_{3}\right)$ and $\left(u_{1}, u_{2}, u_{4}\right)$ is cyclic by verifying, with the assistance of Figure 2.2 , that (a) both edges $\left(u_{1}, u_{3}\right)$ and $\left(u_{1}, u_{4}\right)$ have to be outgoing from $u_{1}$, and (b) at least one of edges $\left(u_{3}, u_{2}\right)$ and $\left(u_{4}, u_{2}\right)$ is incoming to $u_{2}$, as illustrated by Figure 2.3. Let $F$ be an arbitrary spanning forest of $G[B]$, which clearly has $|B|-k$ 
edges. Each cyclic face contains at most two edges of $F$, and each edge of $F$ is incident to at least one cyclic face. Thus, we have $|B|-k \leq 2 c$.

3. Our algorithm. Let $\ell_{i}$ be a given canonical ordering of the input $n$-node plane triangulation $G$. For each $k=1,2, \ldots, n$, let $v_{k}$ be the node with $\ell_{i}\left(v_{k}\right)=k$ and let $G_{k}$ be the subgraph of $G$ induced by $v_{1}, v_{2}, \ldots, v_{k}$. Clearly, $G_{3}$ is a triangle and $v_{1}, v_{2}$, and $v_{n}$ are the external nodes of $G$. Our algorithm initially produces a visibility representation of $G_{3}$, as shown in Figure 3.1, and then extends that into a visibility representation of $G=G_{n}$ in $n-3$ iterations as follows: For each $k=4,5, \ldots, n$, the $(k-3)$ rd iteration obtains a visibility representation of $G_{k}$ from that of $G_{k-1}$ by

1. extending the visibility representation of $G_{k-1}$ in a greedy manner until the node segment of each neighbor of $v_{k}$ is visible from above, and then

2. placing the shortest possible node segment representing $v_{k}$ from above that yields a visibility representation of $G_{k}$.

For example, if $G$ is as shown in Figure 1.1(a) and $\ell_{i}$ is as specified by the node labels, then the visibility representations for $G_{3}, G_{4}, \ldots, G_{11}$ are as shown in Figure 3.1, and the resulting visibility representation of $G=G_{12}$ is as shown in Figure 1.1(b). The correctness of our algorithm follows from the fact that $\ell_{i}$ is a canonical ordering of $G$, which therefore satisfies Lemma 2.1(1)).

A naive implementation of our algorithm takes $O\left(n^{2}\right)$ time. However, it is not difficult to implement our algorithm to run in $O(n)$ time using basic data structures like doubly linked lists to support $O(1)$-time operations such as determining whether a node segment is visible from above and inserting a new column of grid points. More specifically, one can represent each column of grid points by an object in the data structure. A sequence of consecutive columns can then be linked together through a doubly linked list such that the column to the right (respectively, left) of column $c$ can be accessed by right_col $(c)$ (respectively, left_col $(c)$ ). Clearly, one easily can insert a new column of grid points between columns $c$ and right_col $(c)$ in $O(1)$ time by calling subroutine INSERT_COLUMN $(c)$. For each node $v_{i}$ of $G$, we use left_end $(i)$ (respectively, right_end $(i)$ ) to specify the column that contains the left (respectively, right) end of the node segment for $v_{i}$. We also use left_cover $(i)$ (respectively, right_cover $(i)$ ) to specify how far from the left (respectively, right) the node segment for $v_{i}$ is covered by other node segments from above. Clearly, whether the node segment for $v_{i}$ is visible from above can be determined in $O(1)$ time by the condition left_cover $(i) \neq$ left_col(right_cover $(i)$ ). Let leftmost_nbr( $i$ ) (respectively, rightmost_nbr $(i)$ ) denote the leftmost (respectively, rightmost) neighbor of node $v_{i}$ on the boundary of the external face of $G_{i-1}$. A linear-time implementation of the greedy algorithm is as shown in Figure 3.2.

TheOREM 3.1. Any n-node plane triangulation $G$ with $n>3$ has an $O(n)$-time obtainable visibility representation whose width is at most

1. $\left\lfloor\frac{4 n-9}{3}\right\rfloor$ if $G$ has no degree-three internal nodes;

2. $\left\lfloor\frac{4 n-7}{3}\right\rfloor$ if $G$ has no degree-five internal nodes; or

3. $\left\lfloor\frac{22 n-40}{15}\right\rfloor$ if $G$ is unrestricted.

Proof. By Lemma 2.4 and the fact that a realizer is obtainable in linear time, it suffices to show that the width of the output visibility representation by our algorithm is at most $3 n-8-\sum_{v \in I} \operatorname{score}_{i}(v)$. For each $k=4,5, \ldots, n$, consider the iteration that produces the visibility representation of $G_{k}$. Let $v_{j}$ be any neighbor of $v_{k}$ in $G_{k}$. In the first half of the iteration, if the node segment of $v_{j}$ does not contain any grid point that is visible from above, then a new column of grid points is inserted 


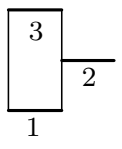

$G_{3}$

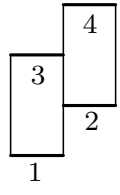

$G_{4}$

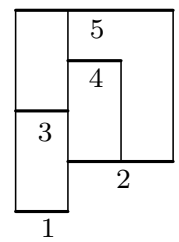

$G_{5}$

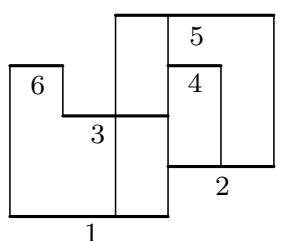

$G_{6}$

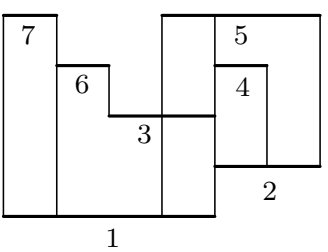

$G_{7}$

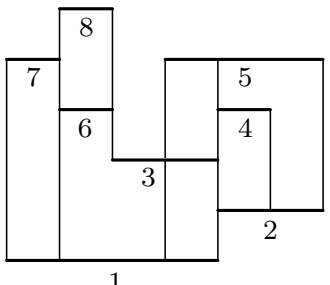

$G_{8}$

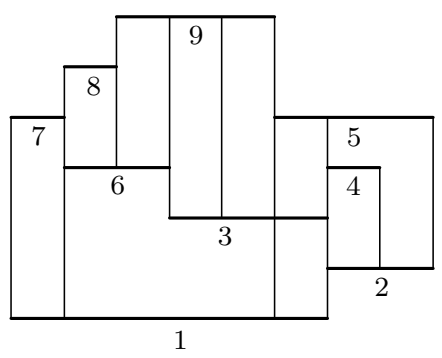

$G_{9}$

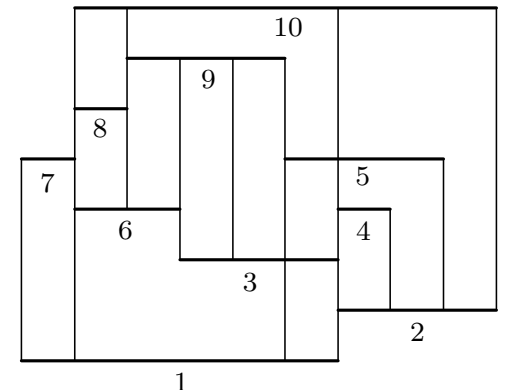

$G_{10}$

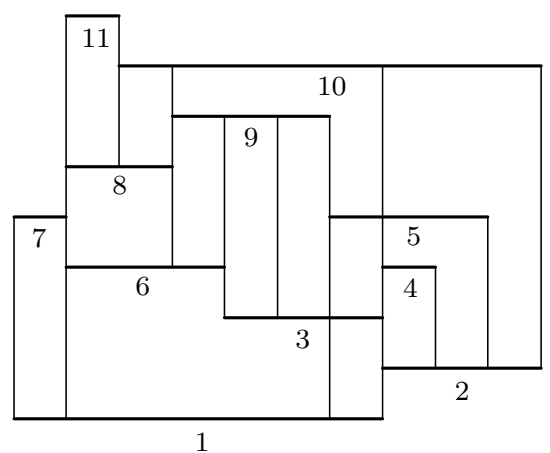

$G_{11}$

FIG. 3.1. The intermediate steps of our algorithm for obtaining the visibility representation for the plane triangulation shown in Figure 1.1(a) with respect to the canonical ordering specified by its node labels.

to ensure that the node segment for $v_{j}$ is visible from above; otherwise, the number of grid points on the node segment of $v_{j}$ that are visible from above stays the same. In the second half of the iteration, if $v_{k}$ is the neighbor of $v_{j}$ with the largest index, then the node segment of $v_{j}$ can no longer be visible from above for the remaining iterations of our algorithm; otherwise, the number of grid points on the node segment of $v_{j}$ that are visible from above decreases by exactly one. Moreover, the node segment of $v_{k}$ contains at least $\operatorname{deg}_{i}^{-}\left(v_{k}\right)$ grid points that are visible from above in the resulting visibility representation of $G_{k}$. Therefore, in the first half of those $\operatorname{deg}_{i}^{+}\left(v_{k}\right)$ iterations, one for each neighbor $v_{\ell}$ of $v_{k}$ with $\ell>k$, at $\operatorname{most} \operatorname{deg}_{i}^{+}\left(v_{k}\right)-\operatorname{score}_{i}\left(v_{k}\right)$ new columns of grid points are inserted. Note that $n>3$ implies $\operatorname{score}_{i}\left(v_{3}\right) \geq 1$. It follows that the resulting visibility representation for $G_{n}=G$ has width at most $2+(-3+$ $\left.\sum_{k=1}^{n-1} \operatorname{deg}_{i}^{+}\left(v_{k}\right)\right)-\left(1+\sum_{k=3}^{n-1} \operatorname{score}_{i}\left(v_{k}\right)\right) \leq 3 n-8-\sum_{v \in I} \operatorname{score}_{i}(v)$. 


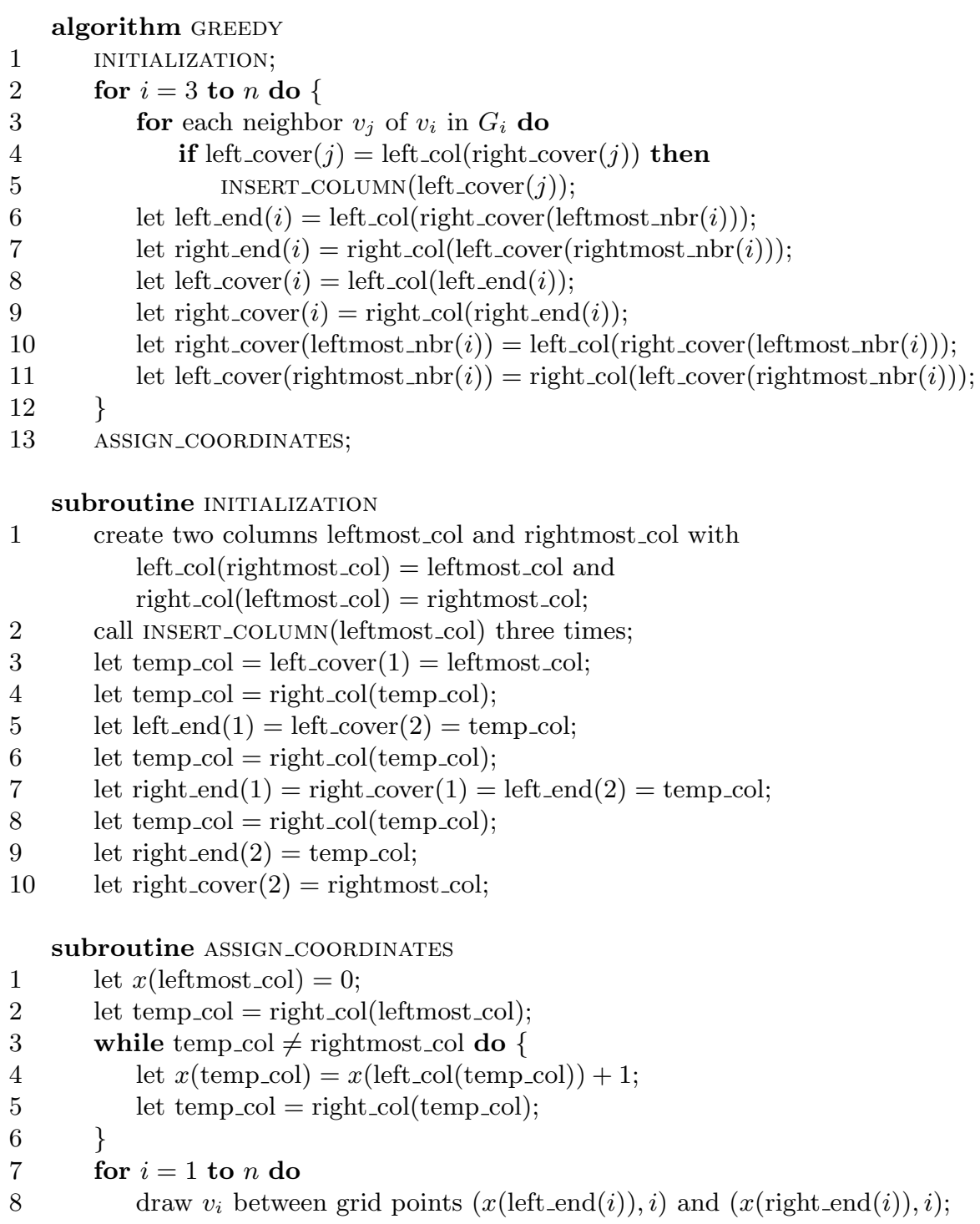

FIG. 3.2. A linear-time implementation of the greedy algorithm.

Remark. As pointed out by an anonymous reviewer, an alternate and possibly quicker way to see the last inequality in the proof of Theorem 3.1 is by the fact that the output of our greedy algorithm is no wider than some constant plus

$$
\begin{aligned}
\sum_{v \in I} \max \left(\operatorname{deg}_{i}^{+}(v)-\operatorname{deg}_{i}^{-}(v), 0\right) & =\sum_{v \in I}\left(\operatorname{deg}_{i}^{+}(v)-\min \left(\operatorname{deg}_{i}^{-}(v), \operatorname{deg}_{i}^{+}(v)\right)\right) \\
& =\sum_{v \in I}\left(\operatorname{deg}_{i}^{+}(v)-\operatorname{score}_{i}(v)\right)
\end{aligned}
$$




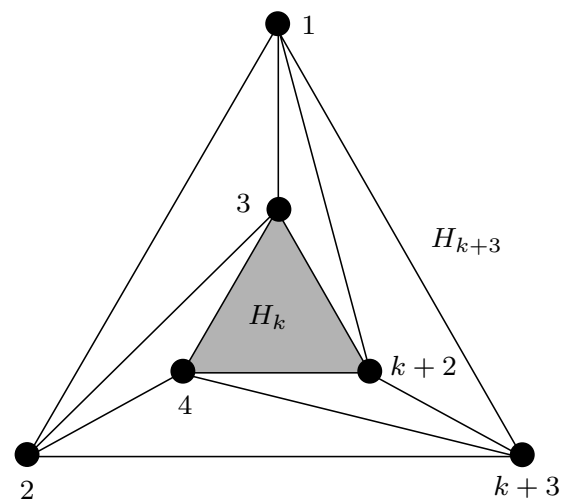

(a)

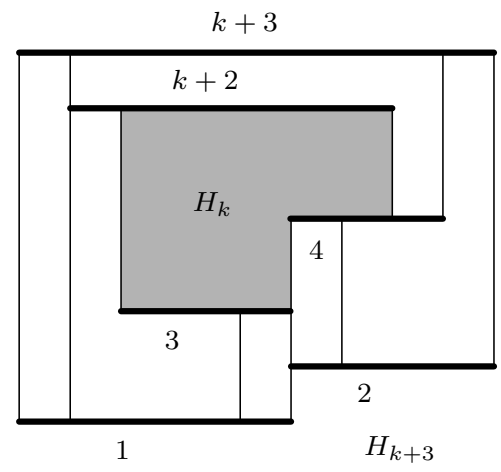

(b)

FIG. 3.3. An example showing that our analysis on the required width is almost tight.

The following result was first obtained by Kant and He [18, 19] and is based upon their linear-time algorithm for obtaining a canonical ordering $\ell_{i}$ for any $n$-node fourconnected plane triangulation such that $\operatorname{deg}_{i}^{+}(v) \geq 2$ and $\operatorname{deg}_{i}^{-}(v) \geq 2$ hold for $n-4$ out of the $n-3$ internal nodes $v$ of $G$. We can alternately prove the theorem in a much simpler way as follows: According to the proof of Theorem 3.1, the width of the output visibility representation by our algorithm is at most $3 n-8-\sum_{v \in I} \operatorname{score}_{i}(v) \leq n-1$.

THEOREM 3.2 (see $[18,19]$ ). If $G$ is an n-node four-connected plane triangulation, then there is an $O(n)$-time obtainable visibility representation for $G$ whose width is at most $n-1$.

4. Near tightness of our analysis. The following lemma shows that our analysis on the required width is almost tight.

LEMma 4.1. For any $n>3$, there exists an $n$-node plane triangulation $H_{n}$ such that any visibility representation of $H_{n}$ obtained by our algorithm with respect to any canonical ordering of $H_{n}$ has width at least $\left\lfloor\frac{4 n-9}{3}\right\rfloor$.

Proof. We prove the lemma by induction on $n$. Let $H_{3}$ (respectively, $H_{4}$ and $H_{5}$ ) be a plane triangulation with 3 (respectively, 4 and 5) nodes. Clearly, any visibility representation of $H_{3}$ (respectively, $H_{4}$ and $H_{5}$ ) has width at least 2 (respectively, 3 and 4 ), so the lemma holds for $n=3,4,5$. For each index $k \geq 3$, let $H_{k+3}$ be the $(k+3)$-node plane triangulation obtained from $H_{k}$ by adding three new external nodes and triangulating the faces as shown in Figure 3.3(a). By Lemma 2.1(1), if $\ell$ is a canonical ordering of $H_{k+3}$, then the ordering $\ell^{\prime}$ with $\ell^{\prime}(v)=\ell(v)-2$ for each node $v$ of $H_{k}$ remains a canonical ordering of $H_{k}$. As illustrated in Figure 3.3(b), it is not difficult to see that the visibility representation for $H_{k+3}$ produced by our algorithm with respect to any canonical ordering of $\mathrm{H}_{k+3}$ is at least four units wider than that of $H_{k}$ produced by our algorithm with respect to any canonical ordering of $H_{k}$. Therefore, the lemma is proved.

5. Concluding remarks. Very recently, Zhang and He [33] showed a linear-time algorithm that produces a visibility representation with height no more than $\left\lceil\frac{15 n}{16}\right\rceil$. It would be interesting to see if combining their techniques and ours could reduce the worst-case area of a visibility representation to significantly less than $\frac{22 n^{2}}{15}-\Theta(n)$.

Whether our upper bound $\frac{22 n}{15}-\Theta(1)$ on the required width is worst-case optimal remains open. We conjecture that any $n$-node plane graph $G$ admits an $s t$-ordering 
with which the greedy algorithm produces a visibility representation for $G$ that is no wider than $\frac{4 n}{3}+O(1)$. We also believe that the worst-case width could be further reduced to $n+O(1)$ if the plane embedding of the input graph could be altered.

Acknowledgment. We thank the anonymous reviewers for their helpful comments.

\section{REFERENCES}

[1] N. Bonichon, C. Gavoille, And N. Hanusse, An information-theoretic upper bound of planar graphs using triangulation, in Proceedings of the 20th Annual Symposium on Theoretical Aspects of Computer Science, H. Alt and M. Habib, eds., Lecture Notes in Comput. Sci. 2607, Springer-Verlag, Berlin, 2003, pp. 499-510.

[2] N. Bonichon, B. Le SAËc, And M. Mosbah, Wagner's theorem on realizers, in Proceedings of the 29th International Colloquium on Automata, Languages, and Programming, P. Widmayer, F. Triguero, R. Morales, M. Hennessy, S. Eidenbenz, and R. Conejo, eds., Lecture Notes in Comput. Sci. 2380, Springer-Verlag, Berlin, 2002, pp. 1043-1053.

[3] H.-L. Chen, C.-C. Liao, H.-I. Lu, And H.-C. Yen, Some applications of orderly spanning trees in graph drawing, in Proceedings of the 10th International Symposium on Graph Drawing, S. G. Kobourov and M. T. Goodrich, eds., Lecture Notes in Comput. Sci. 2528, Springer-Verlag, Berlin, 2002, pp. 332-343.

[4] Y.-T. ChiAng, C.-C. Lin, And H.-I. Lu, Orderly spanning trees with applications to graph encoding and graph drawing, in Proceedings of the 12th Annual ACM-SIAM Symposium on Discrete Algorithms, ACM, New York, SIAM, Philadelphia, 2001, pp. 506-515.

[5] M. Chrobak and G. Kant, Convex grid drawings of 3-connected planar graphs, Internat. J. Comput. Geom. Appl., 7 (1997), pp. 211-223.

[6] R. C.-N. Chuang, A. Garg, X. He, M.-Y. KaO, And H.-I. Lu, Compact encodings of planar graphs via canonical ordering and multiple parentheses, in Proceedings of the 25th International Colloquium on Automata, Languages, and Programming, K. G. Larsen, S. Skyum, and G. Winskel, eds., Lecture Notes in Comput. Sci. 1443, Springer-Verlag, Berlin, 1998, pp. 118-129.

[7] H. De Fraysseix, P. Ossona de Mendez, and P. Rosenstiehl, On triangle contact graphs, Combin. Probab. Comput., 3 (1994), pp. 233-246.

[8] H. De Fraysseix, J. PaCh, And R. POllack, How to draw a planar graph on a grid, Combinatorica, 10 (1990), pp. 41-51.

[9] G. Di Battista, R. Tamassia, and I. G. Tollis, Constrained visibility representations of graphs, Inform. Process. Lett., 41 (1992), pp. 1-7.

[10] S. Even and R. E. Tarjan, Computing an st-numbering, Theoret. Comput. Sci., 2 (1976), pp. 436-441.

[11] U. Fössmeier, G. Kant, and M. Kaufmann, 2-visibility drawings of planar graphs, in Proceedings of the 4th International Symposium on Graph Drawing, S. North, ed., Lecture Notes in Comput. Sci. 1190, Springer-Verlag, Berlin, 1997, pp. 155-168.

[12] X. HE, On floor-plan of plane graphs, SIAM J. Comput., 28 (1999), pp. 2150-2167.

[13] X. HE, A simple linear time algorithm for proper box rectangular drawings of plane graphs, J. Algorithms, 40 (2001), pp. 82-101.

[14] A. Kanevsky, R. Tamassia, G. Di Battista, and J. Chen, On-line maintenance of the four-connected components of a graph, in Proceedings of the 32nd Annual Symposium on Foundations of Computer Science, IEEE, Piscataway, NJ, 1991, pp. 793-801.

[15] G. Kant, A more compact visibility representation, in Proceedings of the 19th Workshop on Graph-Theoretic Concepts in Computer Science, J. van Leeuwen, ed., Lecture Notes in Comput. Sci. 790, Springer-Verlag, Berlin, 1994, pp. 411-424.

[16] G. KANT, Drawing planar graphs using the canonical ordering, Algorithmica, 16 (1996), pp. 432 .

[17] G. Kant, A more compact visibility representation, Internat. J. Comput. Geom. Appl., 7 (1997), pp. 197-210.

[18] G. KANT AND X. HE, Two algorithms for finding rectangular duals of planar graphs, in Proceedings of the 19th Workshop on Graph-Theoretic Concepts in Computer Science, J. van Leeuwen, ed., Lecture Notes in Comput. Sci. 790, Springer-Verlag, Berlin, 1994, pp. 396-410.

[19] G. Kant And X. He, Regular edge labeling of 4-connected plane graphs and its applications in graph drawing problems, Theoret. Comput. Sci., 172 (1997), pp. 175-193. 
[20] C.-C. LiaO, H.-I. Lu, And H.-C. Yen, Floor-planning via orderly spanning trees, in Proceedings of the 9th International Symposium on Graph Drawing, P. Mutzel, M. Jünger, and S. Leipert, eds., Lecture Notes in Comput. Sci. 2265, Springer-Verlag, Berlin, 2002, pp. $367-377$.

[21] C.-C. Lin, H.-I. Lu, AND I.-F. Sun, Improved compact visibility representation of planar graph via Schnyder's realizer, in Proceedings of the 20th Annual Symposium on Theoretical Aspects of Computer Science, H. Alt and M. Habib, eds., Lecture Notes in Comput. Sci. 2607, Springer-Verlag, Berlin, 2003, pp. 14-25.

[22] H.-I. LU, Improved compact routing tables for planar networks via orderly spanning trees, in Proceedings of the 8th International Conference on Computing and Combinatorics, O. H. Ibarra and L. Zhang, eds., Lecture Notes in Comput. Sci. 2387, Springer-Verlag, Berlin, 2002, pp. 57-66.

[23] C.-I. NAKAno, Planar drawings of plane graphs, IEICE Trans. Inform. Systems, E83-D (2000), pp. 384-391.

[24] J. NummenmaA, Constructing compact rectilinear planar layouts using canonical representation of planar graphs, Theoret. Comput. Sci., 99 (1992), pp. 213-230.

[25] R. OtTEn AND J. van WiJK, Graph representations in interactive layout design, in Proceedings of the IEEE International Symposium on Circuits and Systems, 1978, pp. 914-918.

[26] P. Rosenstiehl And R. E. TARJAn, Rectilinear planar layouts and bipolar orientations of planar graphs, Discrete Comput. Geom., 1 (1986), pp. 343-353.

[27] M. Schlag, F. Luccio, P. Maestrini, D. T. Lee, and C. K. Wong, A visibility problem in VLSI layout compaction, in Advances in Computing Research, vol. 2, F. P. Preparata, ed., JAI Press, Greenwich, CT, 1985, pp. 259-282.

[28] W. Schnyder, Planar graphs and poset dimension, Order, 5 (1989), pp. 323-343.

[29] W. SCHNYDER, Embedding planar graphs on the grid, in Proceedings of the First Annual ACMSIAM Symposium on Discrete Algorithms, ACM, New York, SIAM, Philadelphia, 1990, pp. $138-148$.

[30] R. TAmassia And I. G. Tollis, A unified approach to visibility representations of planar graphs, Discrete Comput. Geom., 1 (1986), pp. 321-341.

[31] R. Tamassia and I. G. Tollis, Planar grid embedding in linear time, IEEE Trans. Circuits Systems, 36 (1989), pp. 1230-1234.

[32] K. Wagner, Bemerkungen zum vierfarbenproblem, Jahresber. Deutsch. Math.-Verein, 46 (1936), pp. 26-32.

[33] H. Zhang AND X. He, Compact visibility representation and straight-line grid embedding of plane graphs, in Proceedings of the 8th International Workshop on Algorithms and Data Structures, F. Dehne, J. R. Sackand, and M. Smid, eds., Lecture Notes in Comput. Sci. 2748, Springer-Verlag, Berlin, 2003, pp. 493-504. 